\title{
LEVEL OF GLYCATION END PRODUCTS AND GALACTIN-3 IN PATIENTS WITH CHRONIC HEART FAILURE AND ATRIAL FIBRILLATION IN DEPENDENCE ON THE AGE AND RENAL FUNCTIONAL
}

\author{
Olexander Kuryata \\ Department of Internal Medicine 2 \\ SI «Dnipropeetrovsk Medical Academy of Health Ministry of Ukraine» \\ 9 Vernadskogo str., Dnipro, Ukraine, 49000 \\ Muhammad Muhammad \\ Department of Internal Medicine 2 \\ SI «Dnipropetrovsk Medical Academy of Health Ministry of Ukraine» \\ 9 Vernadskogo str., Dnipro, Ukraine, 49000 \\ drmuhammad2@gmail.com \\ Olga Mytrokhina \\ Department of Internal Medicine 2 \\ SI «Dnipropetrovsk Medical Academy of Health Ministry of Ukraine» \\ 9 Vernadskogo str., Dnipro, Ukraine, 49000 \\ mos.md.80@gmail.com
}

\begin{abstract}
Aim. To analyze the changes of advanced glycation end products (AGE) and galectin-3, their relationship in patients with chronic heart failure (CHF) and atrial fibrillation (AF), depending on the renal functional and age.

Material and methods. 30 patients with CHF II-III FC according to the classification of the New York Heart Association (NYHA) with preserved systolic function were examined (EF>45 \%, mean 58.73 [45.38, 88.00] \%), mean age 66.86 [46.00, 85.00] years who were on treatment in the Regional Hospital after

I. I. Mechnikov. Men accounted for $60 \%$ (18 people), women - $40 \%$ (12 people). All patients were with AF: 17 (56.7 \%) with a permanent form, $13(43.3 \%)$ - with persistent. The serum creatinine level was determined and the GFR was calculated by CKD-EPI. The level of galectin-3 in the blood was determined by immunofermentive analis using the "Human Galectin-3 Platinum ELISA" kit (GmbH, Austria) on the Stat Fax 2100 (USA) immunofermentive plate analyzer. The fluorescent AGEs in plasma were analysed by quantitative autofluorescence (fluorimeter Hoefer DQ 2000, USA) with fixed spectrum of excitation at $460 \mathrm{~nm}$ with $20 \%$ quinine solution as a standard with results expressed with conversion to glycated albumin.

Results. AGE (mean $1.579[0.884,2.796])$ and galectin-3 (mean $8.542[2.72,17.73])$ levels increased in $83.3 \%$ (25 patients) and $23.3 \%$ (7 patients) respectively. The level of galectin-3 increased with age (by $33.18 \%$, p $<0.05$ ), while the level of AGE did not have significant changes. Levels of AGE and galectin-3 increased with a decrease in GFR (by $18.9 \%, \mathrm{p}<0.05$ and $18.44 \%$, $\mathrm{p}<0.05$, respectively). The level of AGE and serum creatinine correlated to GFR ( $r=0.25, p<0.05$ and $r=-0.22, p<0.05$, respectively), while the level of galectin-3 correlated to GFR ( $r=-0.16, \mathrm{p}<0.05$ ). Intake of irbesartan reduced the level of galectin-3 (by $21.66 \%)$ and AGE (by $4.9 \%$ ). The level of serum creatinine was decreased (by $4.89 \%$ ) and GFR was increased (by $6.3 \%$ ) compared with the intake of ACE inhibitors (by $2.9 \%$ and $1.02 \%$, respectively).

Discussion. The results showed changes in AGE with a decrease in GFR in patients with CHF and AF, which makes it possible to consider AGE, as a marker of cardiorenal syndrome and makes it promising to further study it. Galectin-3 showed itself not only as a diagnostic marker, but also as a dynamic (positive effect of using irbesartan). Irbesartan had comparable clinical efficacy with ACE inhibitors in patients with $\mathrm{CHF}$ and AF.

Conclusions. Among patients with CHF and AF, the level of AGE was increased $83.3 \%$ of patients, galectin-3 - in $23.3 \%$. Depending on the age, priority was given to galectin-3 (an increase of $33.18 \%, \mathrm{p}<0.05$ ). The levels of AGE and galectin-3 depends of GFR and were increased (by $18.9 \%$ and $18.44 \%$, p $<0.05$ respectively). The use of irbesartan showed a more significant positive effect on the level of galectin-3 and AGE with GFR in the range $<60 \geq 30 \mathrm{ml} / \mathrm{min} / 1.73 \mathrm{~m}^{2}$.
\end{abstract}

Keywords: glycation end products, galectin-3, glomerular filtration rate, chronic heart failure, atrial fibrillation. 


\section{Introduction}

Chronic cardiovascular diseases (CVD) and chro kidney diseases (KD) are closely interrelated, and they share common risk factors. In the context of CVD and KD, interest in post-translational modifications (PTMs) of proteins has significantly increased. The analysis of PTMs can be useful in identifying mechanisms that play a role in the genesis and/or progression of CVD and $\mathrm{KD}$, since proteins are constantly exposed to various plasma and tissue components under different pathophysiological conditions [1].

Glycation is the main cause of spontaneous disruption of the structure of intracellular and extracellular proteins of different physiological systems. In $0.1-0.2 \%$ of cases, glycation passes through the residues of lysine and arginine [2]. In some areas where the metabolism of proteins is limited (for example, in the lens of the eye), the degree of glycation may increase in 10 times [2]. There are many products of the addition of glucose to proteins of tissues and body fluids in vivo. The earliest product of the addition of glucose to the protein is Ne-fructosyl-lysine (PL), with the slow degradation of which various advanced glycation end products (AGE) are formed. Dicarbonyl compounds of endogenous origin, as well as glyoxal, methylglyoxal and 3-deoxyglucosone have an expressed ability to glycate proteins. They are formed by the degradation of glycated proteins, intermediate metabolites of glycolysis and lipid peroxidation. Dicarbonyl compounds directly react with proteins to form AGE [2]. In the largest amount, hydroimidazolones are formed as the final glycation products, which are derivatives of arginine residues modified by glyoxal, methylglyoxal and 3-deoxyglucosone (3-DG), -N $\delta$ - (5-hydro-4-imidazolon-2-yl) ornithine G-H1), N $\delta$-(5-hydro-5-methyl-4-imidazolon-2-yl)ornithine (MG-H1) and N $\delta$-(5-hydro-5-(2,3,4-trihydroxybutyl) 4-imidazolon-2-yl)ornithine and related structural isomers (3DG-H). Other widely studied AGEs are $\mathrm{N} \delta$-carboxymethyl-lysine $(\mathrm{CML})$ and $\mathrm{N} \delta$-carboxyethyl-lysine $(\mathrm{CEL})$, as well as derivatives of cross-linking proteins - pentosidine and glucosezane [2].

Initially, glycation was considered a reaction of post-translational modification of proteins, primarily extracellular ones. It was assumed, that AGE slowly accumulates in the body throughout life, and the concentrations of AGE reflect the process of accumulation of attachment products. However, this is only true for chemically stable AGEs formed from long-lived proteins, while in physiological conditions PL and some other AGE (in particular, hydroimidazolones) have a relatively short half-life (2-6 weeks) and can be formed from intracellular and short-lived extracellular proteins. In the degradation of proteins with glycation altered by structure, glycation products are released [2]. In vivo peptides of low molecular weight are found in small concentrations in the blood plasma and in urine, and they can also be glycated. The products of glycation of proteins and peptides are called "remnants of glycation products", which corresponds to the nomenclature of amino acid residues in the composition of proteins. Free glycation products are glycated amino acids formed with complete proteolysis of glycated proteins and glycation of amino acids. They are excreted in the urine [2].

Glycation is the main cause of spontaneous breakdown of the structure of proteins in the body. AGE stimulates various pro-inflammatory and signaling metabolic pathways, and their excessive accumulation in the body is considered as one of the main factors in the pathogenesis of cardiovascular diseases [3]. The participation of AGE in the pathogenesis of vascular complications is confirmed by the following experimental data:

1) accumulation of AGE residues at the sites of vascular injuries;

2) against the background of a change in the structure of vascular cell proteins under the influence of AGE in vitro, cell dysfunction is observed;

3) inhibitors of AGE formation suppress the development of vascular complications (according to the data of the conducted experiments and clinical studies).

Atrial fibrillation (AF) is one of the most common arrhythmias, a prevalence that increases as the population age growth [4]. Molecular mechanisms involved in atrial remodeling are not fully understood, but it is known that the pro-fibrosis and proinflammatory mechanisms increase the risk of AF. One such mechanism that occurs with aging involves the non-enzymatic binding of proteins and lipids leading to the formation of AGE [4]. AGE can play a role in the pathophysiology of PL through their interaction with cellular receptors for AGE (RAGE), which causes inflammation 
and fibrosis through successive activation of the nuclear factor kappa B and increased expression of cytokines and adhesion molecules. Recent studies have shown that the interaction of AGE and RAGE can contribute to the onset of inflammation and oxidative stress [4]. These processes may also participate in the pathogenesis of $\mathrm{AF}$, but their role remains debatable.

Various proteins are considered as specific receptors for AGE-modified proteins. Among the receptors located on the cell surface, they include the receptor of phagocytes, the receptor of the advanced glycation end products (RAGE) and galectin-3. Other AGE-binding proteins are localized in the cytoplasm and, possibly, they are not receptors for AGE [2]. Galectin-3 is considered as an AGE receptor, as evidenced by the ability of high-glycated albumin to interact with recombinant galectin-3, as well as the specific capture of galectin-3 by macrophages of RAW mice by the same ligand [2]. In vitro galectin-3 provides protection for mesangial cells, prevents the attack of infiltrating macrophages and cytotoxic effects of TGF- $\beta$, affects the interaction of proteoglycans during angiogenesis, and also has other intracellular and extracellular effects in vivo [2]. Indeed, galectin-3 has a pro-inflammatory effect: in the case of nephropathy, galectin-3+ infiltrates macrophages in the distal tubules and glomerulus of the kidneys, and the nature of this infiltration correlates with the severity of nephropathy $[2,5]$. Thus, proteins classified as AGE receptors are involved in the pathogenesis of vascular complications, but it is not completely known whether the functional activity of these receptors is enhanced under the influence of AGE-modified proteins in vivo [2]. It should be noted, that galectin-3 promotes the migration of macrophages, the proliferation of fibroblasts and the synthesis of collagen in cardiomyocytes [6, 7]. Increased expression of galectin-3 causes remodeling of the left ventricle (LV), which is accompanied by cardiac dysfunction and a decrease in the contractile function of the heart $[8,9]$.

It is known that the accumulation of AGE is one of the causes of CHF, nephropathy. Studies have shown that angiotensin II receptor blockers (ARBs) can reduce the ability to bind AGE to hypertensive patients. In this regard, our interest is the fact of studying glycation products in patients with $\mathrm{CHF}$ and $\mathrm{AF}$, depending on the renal functional.

\section{Aim of the research}

To analyze the changes of advanced glycation end products (AGE) and galectin-3, their relationship in patients with chronic heart failure (CHF) and atrial fibrillation (AF), depending on the renal functional and age.

\section{Materials and methods}

30 patients with CHF II-III FC according to the classification of the New York Heart Association (NYHA) with preserved systolic function were examined ( $\mathrm{EF}>45 \%$, mean 58.73 [45.38, $88.00] \%$, mean age 66.86 [46.00, 85.00] years who were on treatment in the Regional Hospital a fter I. I. Mechnikov. The study was conducted from December 2016 to December 2017. The observation period was 3 months. Among patients were $60 \%$ (18 people) men and $40 \%$ (12 people) women. All patients were with AF: $17(56.7 \%)$ - with a permanent form, 13 (43.3\%) - with persistent.

Verification of the diagnosis of CHF and AF was conducted according to the recommendations of the European Society of Cardiology [10, 11].

Inclusion criteria were the presence of $\mathrm{CHF}$ with a preserved ejection fraction (EF>45\%) under conditions of stably selected drug therapy, AF, glomerular filtration rate (GFR) $<90 \geq 30 \mathrm{ml} / \mathrm{min} / 1.73 \mathrm{~m}^{2}$ and informed consent of the patient.

Exclusion criteria were patients with CHF IV FC and acute heart failure; acute coronary syndrome; myocardial infarction and stroke up to 6 months; significant valvular heart disease, GFR $<30 \mathrm{ml} / \mathrm{min} / 1.73 \mathrm{~m}^{2}$.

For clinical evaluation of renal function, serum creatinine was determined using colorimetric methods and the GFR was calculated by CKD-EPI according to the recommendations of the American National Federation of the Kidneys [12].

All patients were assessed with galectin-3 blood levels by immunofermentive analis using the "Human Galectin-3 Platinum ELISA" kit (GmbH, Austria) on the Stat Fax 2100 (USA) immunoassay plate analyzer. The base level was taken as $0 \mathrm{ng} / \mathrm{ml}$. 
The fluorescent AGEs in plasma were analysed by quantitative autofluorescence (fluorimeter Hoefer DQ 2000, USA) with fixed spectrum of excitation at $460 \mathrm{~nm}$ with $20 \%$ quinine solution as a standard with results expressed with conversion to glycated albumin.

15 patients (the main group) who prior to inclusion in the study did not received ARBs or had a poor tolerance of ACE inhibitors, irbesartan was used at a dose of $150 \mathrm{mg}$ orally once a day, with an insufficient antihypertensive effect $(\mathrm{BP}>140 / 90 \mathrm{~mm} \mathrm{Hg}$ ) after 1 week the dose of the drug was increased to $300 \mathrm{mg}$ per day. 15 patients (comparison group) continued to receive therapy with ACE inhibitors.

For statistical analysis of data was used STATISTICA v.6.1 (Statsoft Inc., USA) license program. Categorical variables were expressed as frequencies and percentages. With regard to continuous variables, the assumption of normality was tested with the Kolmogorov-Smirnov test. The variables that follow a normal distribution were expressed as mean \pm standard deviation. The remaining variables were expressed as median and interquartile range. [13].

\section{Results}

During the examination, arterial hypertension (AH) of stage II was registered in $19(63.3 \%)$, stage III - in 5 (16.6 \%) patients; AH of I degree - in 3 (10\%), II - in 18 (60\%), III - in $3(10 \%)$ patients $5(16.7 \%)$ - had a history of myocardial infarction (more than 6 month) (Table 1).

Table 1

Characteristics of patients with $\mathrm{CHF}$ and $\mathrm{AF}(\mathrm{n}=30)$

\begin{tabular}{cc}
\hline Indicators & Patients with CHF and AF \\
\hline Blood pressure $(\mathrm{BP}), \mathrm{mm} \mathrm{Hg}$ & \\
systolic $(\mathrm{Me}[25 \% ; 75 \%])$ & $141,50[100 ; 180]$ \\
diastolic $(\mathrm{Me}[25 \% ; 75 \%])$ & $87,53[70 ; 112]$ \\
Heart rate, contr. per min. $(\mathrm{Me}[25 \% ; 75 \%])$ & $77,00[65 ; 98]$ \\
EF, \% (Me $[25 \% ; 75 \%])$ & $58,73[45.38 ; 88]$ \\
Body mass index (BMI), kg/m² & $29,77[23.55 ; 40.9]$ \\
(Me $[25 \% ; 75 \%])$ & \\
Arterial hypertension, $\mathrm{n}(\%)$ & $19(63.3 \%)$ \\
II stage & $5(16.6 \%)$ \\
III stage & $3(10 \%)$ \\
I degree & $18(60 \%)$ \\
II degree & $3(10 \%)$ \\
III degree & $5(16.7 \%)$
\end{tabular}

Patients were used treatment according to recommendations [10, 11] (Table 2).

Table 2

Drug therapy of patients with $\mathrm{CHF}$ and AF

\begin{tabular}{ccc}
\hline Indicators & $\begin{array}{c}\text { Main group } \\
(\mathbf{n}=\mathbf{1 5})\end{array}$ & $\begin{array}{c}\text { Comparison group } \\
(\mathbf{n}=\mathbf{1 5})\end{array}$ \\
\hline$\beta$-blockers, \% & $11(73.3 \%)$ & $12(80 \%)$ \\
Diuretics, $\%$ & $9(60 \%)$ & $6(40 \%)$ \\
Calcium antagonists, $\%$ & $3(20 \%)$ & $2(13.3 \%)$ \\
Aspirin, \% & $12(80 \%)$ & $12(80 \%)$ \\
Statins, $\%$ & $6(40 \%)$ & $8(53.3 \%)$
\end{tabular}

Among the examined, $83.3 \%$ of patients (25 people) had an elevated level of AGE (mean $1.579[0.884,2.796])$ (normal level $-0.9-1.1 \mathrm{mg} / \mathrm{ml}$ ). The level of galectin-3 was increased in 
7 patients $(23.3 \%$ ) (normal level $<12 \mathrm{ng} / \mathrm{ml}$ ), mean -8.542 [2.72; 17.73], which corresponds to normal values.

The obtained results showed that an increase in the FC of CHF was not accompanied by a significant change in the levels of galectin-3 and AGE, but a tendency toward an increase in the indices of galectin-3 was noted, which corresponds to the literature data (Table 3).

Table 3

The level of AGE and galectin-3, depending of the FC in patients with CHF and AF

\begin{tabular}{|c|c|c|c|}
\hline & \multicolumn{3}{|c|}{$\mathrm{CHF}$ and $\mathrm{AF}(\mathrm{n}=30)$} \\
\hline & & & FC III \\
\hline Galectin-3 & \multicolumn{2}{|c|}{$\begin{array}{c}7.987 \\
{[3.11 ; 16.16]}\end{array}$} & $\begin{array}{c}8.746 \\
{[2.72 ; 17.73]}\end{array}$ \\
\hline AGE & \multicolumn{2}{|c|}{$\begin{array}{c}1.641 \\
{[1.088 ; 2.796]}\end{array}$} & $\begin{array}{c}1.556 \\
{[0.884 ; 2.570]}\end{array}$ \\
\hline \multicolumn{4}{|c|}{$\begin{array}{l}\text { It is known that in the development and progression of CHF plays an important role such a } \\
\text { factor as age. In connection with this, the study of markers that determine the features of the course } \\
\text { of CHF in different age groups is of great importance (Table 4). }\end{array}$} \\
\hline \multicolumn{4}{|l|}{ Table 4} \\
\hline & \multicolumn{3}{|c|}{ CHF and $\mathrm{AF}(\mathrm{n}=30)$} \\
\hline & 45-59 ages & 60-74 ages & $75-85$ ages \\
\hline Galectin-3 & $\begin{array}{c}6.875 \\
{[3.85 ; 12.8]}\end{array}$ & $\begin{array}{c}8.293 \\
{[2.72 ; 16.16]}\end{array}$ & $\begin{array}{c}10.290 * \\
{[3.87 ; 17.73]}\end{array}$ \\
\hline AGE & $\begin{array}{c}1.657 \\
{[1.314 ; 2.120]}\end{array}$ & $\begin{array}{c}1.581 \\
{[0.884 ; 2.796]}\end{array}$ & $\begin{array}{c}1.516 \\
{[1.064 ; 2.028]}\end{array}$ \\
\hline
\end{tabular}

Note: *- the reliability of differences compared with the group of 45-59 years, $p<0.05$

The analysis showed that the level of galectin-3 increases with age (by $33.18 \%, \mathrm{p}<0.05$ ), while the AGE level has no significant changes.

It is believed that the active accumulation of AGE in the blood is due to their reduced renal clearance. To clarify the role of AGE and galectin-3 in the development and progression of renal dysfunction, patients were further analysed depending on the level of GFR (Table 5). It should be noted that 6 patients $(20 \%)$ had normal renal function.

Table 5

The level of AGE and galectin-3 depending of GFR in patients with CHF and AF

\begin{tabular}{ccc}
\hline & $\mathbf{G F R}<\mathbf{9 0} \geq \mathbf{6 0} \mathbf{~ m l} / \mathbf{m i n} . \mathbf{1}, \mathbf{7 3} \mathbf{m}^{\mathbf{2}} \mathbf{( n = 1 2 )}$ & $\mathbf{G F R}<\mathbf{6 0} \geq \mathbf{3 0} \mathbf{~ m l} / \mathbf{m i n} . \mathbf{1} \mathbf{1 7 3} \mathbf{m}^{\mathbf{2}}(\mathbf{n}=\mathbf{1 2})$ \\
\hline Galectin-3 & 7.670 & 9.405 \\
AGE & {$[2.72 ; 17.73]$} & {$[4.1 ; 16.2]^{*}$} \\
& {$[0.884 ; 1.878]$} & 1,781 \\
& & {$[0.952 ; 2.796]^{*}$}
\end{tabular}

Note: * the reliability of the differences compared to the GFR group $<90 \geq 60 \mathrm{ml} / \mathrm{min} / 1.73 \mathrm{~m}^{2}, p<0.05$

The results showed that with a decrease in GFR there was an increase in the level of both AGE (by $18.9 \%, p<0.05$ ) and galectin-3 (by $18.44 \%$, p < 0.05 ). Correlation analysis revealed a close relationship between the level of AGE, serum creatinine and GFR $(r=0.25, p<0.05$ and $r=-0.22$, $\mathrm{p}<0.05$, respectively), while the level of galectin-3 correlated only with GFR $(r=-0.16, p<0.05)$.

After 3 months of treatment, the level of galectin-3 (by $21.66 \%$ ) and AGE (by $4.9 \%$ ) was decreased (Table 6). 
Table 6

Effect of therapy on the level of galectin-3 and AGE in patients with CHF and AF

\begin{tabular}{ccccc}
\hline & \multicolumn{2}{c}{ Main group } & \multicolumn{2}{c}{ Comparison group } \\
\cline { 2 - 5 } & Before the treatment & After the treatment & Before the treatment & After the treatment \\
\hline Galectin-3 & 10.161 & 7.960 & 8.271 & 5.850 \\
AGE & {$[4.1 ; 17.73]$} & {$[3.9 ; 14.7]$} & {$[3.85 ; 16.16]$} & {$[2.94 ; 13.48]$} \\
& 1.641 & 1.559 & 1.516 & 1.537 \\
& {$[0.884 ; 2.796]$} & {$[0.886 ; 2.890]$} & {$[1.078 ; 2.120]$} & {$[1.050 ; 2.340]$}
\end{tabular}

Against the backdrop of treatment with irbesartan, a decrease in the level of serum creatinine (by $4.89 \%$ ) and an increase in GFR (by $6.3 \%$ ) compared with the use of ACE inhibitors (by $2.9 \%$ and $1.02 \%$, respectively) were detected.

The inclusion of irbesartan contributed to a significant decrease in the level of galectin-3 among patients with GFR $<60 \geq 30 \mathrm{ml} / \mathrm{min} / 1.73 \mathrm{~m}^{2}(\mathrm{p}<0.05)$ compared with $\mathrm{GFR}<90 \geq 60 \mathrm{ml} / \mathrm{min} /$ $1.73 \mathrm{~m}^{2}$ (Fig. 1).

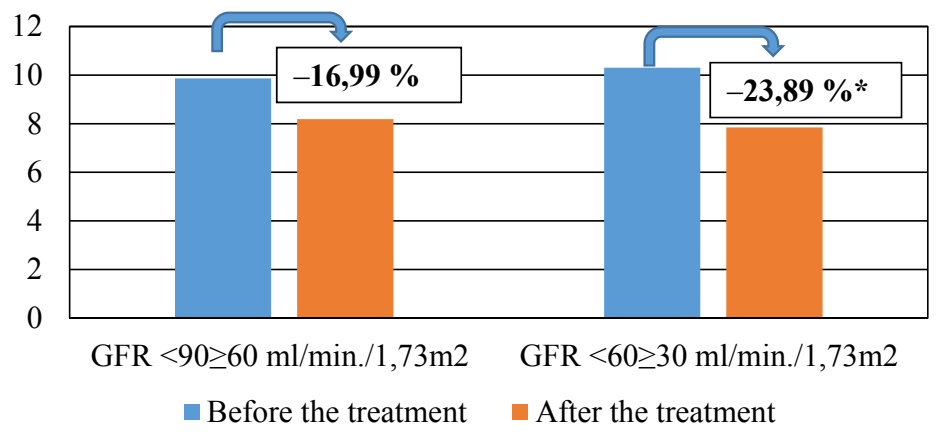

Fig. 1. Dynamics of the level of galectin-3 in patients with $\mathrm{CHF}$ and AF who intake irbesartan:

* - the reliability of differences compared with the baseline, $\mathrm{p}<0.05$

Against the backdrop of treatment, there was a trend towards a decrease in the level of AGE among patients with GFR $<60 \geq 30 \mathrm{ml} / \mathrm{min} / 1.73 \mathrm{~m}^{2}$, while there were no significant changes in patients with $\mathrm{CHF}$ and AF with GFR $<90 \geq 60 \mathrm{ml} / \mathrm{min} / 1,73 \mathrm{~m}^{2}$ (Fig. 2).

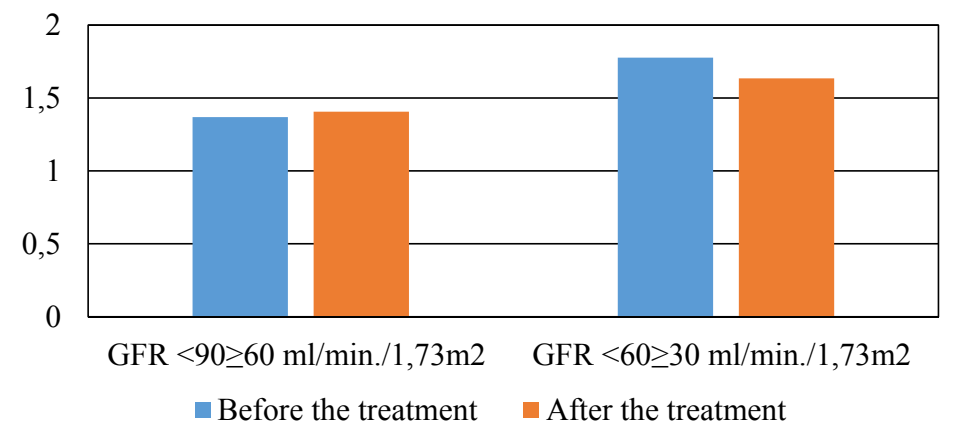

Fig. 2. Dynamics of AGE level in patients with $\mathrm{CHF}$ and $\mathrm{AF}$ who intake irbesartan

\section{Discussion}

Accumulation of AGEs can be explained by certain cardiovascular changes, such as vessel stiffness, diastolic dysfunction and endothelial dysfunction. From the reports of Semba et al., it follows that a high concentration of AGE may be a risk factor for cardiovascular disease, but additional evidence is needed to confirm this statement $[14,15]$.

AGEs can promote the development of chronic heart failure (CHF) in two ways. First, AGEs affect the physiological properties of proteins in the extracellular matrix, creating crosslinks. Secondly, AGEs causes multiple changes in blood vessels and myocardium through 
interaction with AGEs receptors. AGEs can cause diastolic, systolic and vascular dysfunction through these pathways. Subsequently, these disorders can lead to the development and progression of CHF [16].

Strong evidence has been obtained in favour of the fact that the residues of AGE accumulate in the zones of vascular lesions, namely in the glomeruli of the kidneys [2]. At the same time, AGE-modified proteins break down as a result of cellular proteolysis with the formation of free products of AGE and are excreted in the urine, and therefore, in the presence of uremia is noted their active accumulation. Several mechanisms underlying the renal dysfunction have been proposed, for example, reduced renal perfusion, atherosclerosis and inflammation, endothelial dysfunction and neurohormonal activation [2]. Another possible mechanism may be an increase in AGEs. Accumulation of AGE in patients with renal dysfunction can occur in two ways. First, the increase in accumulation of AGE is due to a decrease in the clearance of the AGE decomposition products. After modification or degradation in the proximal glomeruli, AGEs are ultimately purged in urine [17]. Patients with renal dysfunction have a reduced clearance and, thus, occurs accumulation of AGE. Secondly, oxidative stress is increased in patients with renal dysfunction, which also leads to an increase in AGE formation [17]. Thus, it can be assumed that AGEs are a possible mechanism underlying the renal dysfunction. However, while the concentration of AGE circulation and their potential toxicity have been extensively studied in renal failure, there is no information available in patients with CHF.

For more than 10 years, the problem of cardiovascular syndrome has been discussed in the world. A number of studies have shown the adverse effect of decreased renal function on the course and prognosis of CHF. To date, a steady trend towards the introduction in the world of unified protocols for the treatment of cardiovascular diseases has led to positive results: improving the quality of treatment of patients and reducing mortality. However, the uncertainty of specific measures for the treatment of patients with CHF with preserved ejection fraction (EF $>45 \%)$ and lack of satisfactory results induce to search for new directions of treatment and determine the possibilities of existing methods of drug therapy [18].

In the recommendations of the American College of Cardiology (ACC) and the American Heart Association (AHA) in 2017 for the treatment of patients with CHF, it is recommended to achieve low blood pressure $(130 / 80 \mathrm{~mm} \mathrm{Hg})$, which in turn raises the issue of whiter hard control of blood pressure. Particular attention in the recommendations is given to the use of angiotensin II receptor blockers [19]. There is an opinion that the ARBs have a nephroprotective effect. However, the CHARM study showed that the use of candesartan is ineffective in patients with impaired renal function. While another study IRMA 2, using irbesartan, it was noted that the drug contributed to a reduction in the occurrence of new cases of nephropathy and dialysis, and also reduced the progression of the disease [20].

\section{Conclusions}

1. Among the examined, the elevated level of AGE was $83.3 \%$ of patients, galectin- $3-23.3 \%$.

2. Galectin-3, unlike AGE, clearly varies depending on age (an increase of $33.18 \%$, $\mathrm{p}<0.05$ ) and GFR (an increase of $18.44 \%, \mathrm{p}<0.05$ ), that is has a greater impact range than AGE. However, AGE varies depending on GFR in patients with CRF (an increase of $18.9 \%, p<0.05$ ), which may indicate a real damage to the clearance model and the role of glycosylation in damage to kidney function.

3. Galectin-3 is a more dynamic factor, since a positive response was noted against the background of irbesartan, in contrast to AGE. Thus, it can be said that AGE is a marker that reflects the manifestations of cardiorenal syndrome, and galectin-3 is associated with age and dynamics of CHF itself.

\section{References}

[1] Gajjala, P. R., Fliser, D., Speer, T., Jankowski, V., Jankowski, J. (2015). Emerging role of post-translational modifications in chronic kidney disease and cardiovascular disease. Nephrology Dialysis Transplantation, 30 (11), 1814-1824. doi: 10.1093/ndt/gfv048 
[2] Ahmed, N., Thornalley, P. J. (2009). The role of advanced glycation end products in the pathogenesis of diabetes complications. RMJ, 9, 642-650.

[3] Spasov, A. A., Rashchenko, A. I., Brigadirova, A. A. (2016). Optimization of method in vitro for re-glycation activity of compounds investigation. VolgSMU, 1 (57), 30-32.

[4] Lancefield, T. F., Patel, S. K., Freeman, M., Velkoska, E., Wai, B., Srivastava, P. M. et. al. (2016). The Receptor for Advanced Glycation End Products (RAGE) Is Associated with Persistent Atrial Fibrillation. PLOS ONE, 11 (9), e0161715. doi: 10.1371/journal.pone.0161715

[5] Kikuchi, Y., Kobayashi, S., Hemmi, N., Ikee, R., Hyodo, N., Saigusa, T. et. al. (2004). Galectin-3-positive cell infiltration in human diabetic nephropathy. Nephrology Dialysis Transplantation, 19 (3), 602-607. doi: 10.1093/ndt/gfg603

[6] Tseluiko, V. I., Vashakidze, Z. S., Motylevskaya, T. V., Opolonskaya, N. A. (2012). Galectin-3 in patients with atrial fibrillation. Ukrainian Cardiology Journal, 3, 45-49.

[7] Gyamdzhan, K. A., Drapkina, O. M., Maksimov, M. L. (2014). Galectin-3: clinical and prognostic value in patients with chronic heart failure. Heart failure, 82 (1), 51-55.

[8] DeFilippi, C. R., Felker, G. M. (2010). Galectin-3 in Heart Failure - Linking Fibrosis, Remodelling and Progression. European Cardiology Review, 6 (2), 33-36. doi: 10.15420/ecr.2010.6.2.33

[9] De Boer, R. A., Yu, L., van Veldhuisen, D. J. (2010). Galectin-3 in Cardiac Remodeling and Heart Failure. Current Heart Failure Reports, 7 (1), 1-8. doi: 10.1007/s11897-010-0004-x

[10] Ponikowski, P., Voors, A. A., Anker, S. D., Bueno, H., Cleland, J. G. F., Coats, A. J. S. et. al. (2016). 2016 ESC Guidelines for the diagnosis and treatment of acute and chronic heart failure. European Heart Journal, 37 (27), 2129-2200. doi: 10.1093/eurheartj/ehw128

[11] Kirchhof, P., Benussi, S., Kotecha, D., Ahlsson, A., Atar, D., Casadei, B. et. al. (2016). 2016 ESC Guidelines for the management of atrial fibrillation developed in collaboration with EACTS. European Heart Journal, 37 (38), 2893-2962. doi: 10.1093/eurheartj/ehw210

[12] Eknoyan, G., Lameire, N., Eckardt, K.-U. et. al. (2013). KDIGO 2012 Clinical Practice Guideline for the Evaluation and Management of Chronic Kidney Disease. Kidney International Supplements, 3 (1), 5-150.

[13] Rebrova, O. Yu. (2002). Statistical analysis of medical data. The use of the application package statistics. Moscow: MediaSphere, 312.

[14] Luevano-Contreras, C., Chapman-Novakofski, K. (2010). Dietary Advanced Glycation End Products and Aging. Nutrients, 2 (12), 1247-1265. doi: 10.3390/nu2121247

[15] Semba, R. D., Ferrucci, L., Sun, K., Beck, J., Dalal, M., Varadhan, R. et. al. (2009). Advanced glycation end products and their circulating receptors predict cardiovascular disease mortality in older community-dwelling women. Aging Clinical and Experimental Research, 21 (2), 182-190. doi: 10.1007/ bf03325227

[16] Hartog, J. W. L., Voors, A. A., Bakker, S. J. L., Smit, A. J., van Veldhuisen, D. J. (2007). Advanced glycation end-products (AGEs) and heart failure: Pathophysiology and clinical implications. European Journal of Heart Failure, 9 (12), 1146-1155. doi: 10.1016/j.ejheart.2007.09.009

[17] Willemsen, S., Hartog, J. W. L., Heiner-Fokkema, M. R., van Veldhuisen, D. J., Voors, A. A. (2011). Advanced glycation end-products, a pathophysiological pathway in the cardiorenal syndrome. Heart Failure Reviews, 17 (2), 221-228. doi: 10.1007/s10741-010-9225-z

[18] Reznik, E. V., Storozchakov, G. I. (2009). The heart is ill - kidneys suffer: Cardiorenal Syndrome in Patients with Chronic Heart Failure. Therapeutics, 1, 27-35.

[19] Yancy, C. W., Jessup, M., Bozkurt, B., Butler, J., Casey, D. E., Colvin, M. M. et. al. (2017). 2017 ACC/AHA/HFSA Focused Update of the 2013 ACCF/AHA Guideline for the Management of Heart Failure: A Report of the American College of Cardiology/American Heart Association Task Force on Clinical Practice Guidelines and the Heart Failure Society of America. Circulation, 136 (6), e137-e161. doi: 10.1161/ cir.0000000000000509

[20] Fomin, V. V. (2013). Angiotensin II receptor blockers and control of cardiorenal risk: position of irbesartan. Medical advice, 9, 34-38. 